was noted with low doses of prednisone, however, in contrast with the dramatic improvement obtained in the original papers. ${ }^{56}$

On the other hand, synovial biopsy in our case did not show malignant infiltration. Thus it is reasonable to suggest that the rheumatic manifestations belong to a paraneoplastic syndrome, as has been previously reported with other neoplasms.

To our knowledge this is the first description of peripheral synovitis and pitting oedema as an initial manifestation of non-Hodgkin's lymphoma. We would like to emphasise the need to consider the possibility of an underlying malignancy in a patient with these clinical characteristics who is unresponsive to the usual medical treatment.

M R ROLDAN F MARTINEZ Department of Rheumatolog 'Reina Sofia' Hospital Cordoba Spain

J ROMAN A TORRES Department of Hematology 'Reina Sofia' Hospital Cordoba
Spain

Correspondence to: $M^{\mathrm{a}}$ Rosa Roldan Molina, c/Sanchez de Feria 3, 1" 3, 14003 Cordoba, Spain.

1 Reiner R R, Chabner B A, Young R C. Lymphoma presenting in bone: results of histophoma presenting in bone: results of histopathology, staging and

2 Rice D M, Semble E, Ahl E T, Bohrer S P Rothberger A. Primary lymphoma of bone presenting as monoarthritis. $\mathcal{F}$ Rheumatol 1984 11: $851-4$.

3 Haase K K, Dürk H, Baumbach A, Werhmann M. Non-Hodgkin's lymphoma presenting as fnee monoarthritis with poumatol 1990; 17: 1252-4.

4 Dorfman H D, Siegel H L, Perry M C, Oxenhandler R. Non-Hodgkin's lymphoma of the handler $\mathbf{R}$. Non-Hodgkin's lymphoma of the synovium simulating rheumatoid

5 McCarty D J, O'Duffy J D, Pearson L, Hunter J B. Remitting seronegative symmetrical synovitis with pitting edema. $\mathcal{F A M A} 1985 ; 254$ 2763-7.

6 Russell E B, Hunter J B, Pearson L, McCarty D J. Remitting seronegative symmetrica synovitis with pitting edema- 13 additiona cases. F Rheumatol 1990; 17: 633-9.

7 Sheon R P, Kirsner A B, Tangsintanepas P Samad F, Garg M, Finkel R I. Malignancy in Samad F, Garg M, Finkel R I. Malignancy in Gertiatr Soc 1977; 25: 20.

\section{Arthritis and carcinoma}

Sir: We read with interest the paper by Chakravarty and Webley describing two cases of asymptomatic renal cell carcinoma causing an acute monarthritis, previously unreported. They suggested that this occurrence is both rare and underreported. In our experience this is not necessarily the case. We have recently seen two patients whose cases illustrate some interesting similarities.

Patient No 1, a 63 year old man presented to our hospital with a five week history of a painful, swollen knee joint and was unable to bear weight. Clinical examination confirmed an acute monarthritis of the knee joint, with a tense effusion, warmth, and tenderness. $\mathrm{He}$ had been previously treated for a large cel solid temporo-orbital tumour by radiotherapy because the lesion was considered inoperable. The knee joint effusion was aspirated to rule out sepsis. Bacteriology was negative, no crystals were found, but adenocarcinoma cells were seen in abundance. He made an excellent functional recovery after palliative irradiation to the knee.

Patient No 2, a 63 year old woman, was referred to the radiotherapy department of the same hospital with metastatic disease for which no primary tumour had been located despite extensive investigation. She had initially presented with a painful knee of one year's duration. Clinical examination showed an acute monarthritis with a moderate effusion. Isotope bone scan disclosed a hot spot in the upper tibia. She had a synovial biopsy and cytological examination of the synovial fluid. The synovial biopsy sample showed evidence of infiltration with adenocarcinomatous cells as did the synovial fluid

While we agree with the authors that in cases where there is doubt about the cause of a joint effusion, early examination of synovial fluid is important, and may prevent the need for an open or closed bone biopsy, we are of the opinion that the occurrence of malignant joint effusions is not rare but more likely to be underreported TIM IL IDGE Poole Hospital Longfleet Road Poole
5 27B

Dorset BH15 $27 B$

1 Chakravarty K K, Webley M. Monarthritis: an unusual presentation of renal cell carcinoma. Ann Rheum Dis 1992; 51: 681-2.

\section{Angiotensin converting enzyme in rheumatoid arthritis}

Sir: Being interested in vascular endothelial cell transdifferentiation, ${ }^{1}$ I read with interest the instructive articles by Veale et $a l^{2}$ and Goto et $a l^{3}$ about the angiotensin converting enzyme production in rheumatoid arthritis. Although there is no doubt that vascular endothelial cells participate in this process, the role of macrophages remains questionable because the antibodies used for their identification (antigen CD14) are not macrophage specific and cross react with vascular endothelial cells. ${ }^{45}$ Moreover, undifferentiated vascular endothelial cells can transdifferentiate into macrophage-like cells and migrate into the extravascular space.

It may be useful to re-evaluate the role of macrophages in rheumatoid arthritis in the light of the new knowledge about the transdifferentiation of vascular endothelium. ${ }^{6}$ For example, the mesenchymoid transformation cells responsible for joint destruction differentiate into fibroblasts in due course ${ }^{7}$ and are not, therefore, inflammatory cells. McCachren considers, however, any collagenase producing cells immunoreactive with Leu-M3 or HAM56 to be macrophages. ${ }^{8}$ Again, these antibodies are no macrophage specific and cross react with vascular endothelium. 9 10 Mesenchymoid transformation cells remind me of undifferentiated vascular endothelial cells ${ }^{1}$ by thei character and aggressiveness. ${ }^{7}$ The facts that antiangiogenesis suppresses arthritis ${ }^{11}$ and that angiotensin II is an angiogenic factor ${ }^{12}$ are in excellent keeping with that proposition.

JIRI T BERANEK Division of Cardiology Department of Medicine USA

1 Beranek J T. Atherosclerosis: a personal view. Cardiac Chronicle 1991; 5: 1-6.

2 Veale D, Yanni G, Bresnihan B, FitzGerald O. Production of angiotensin converting enzyme by rheumatoid synovial membrane. Ann Rheum Dis 1992; 51: 476-80.

3 Goto $M$, Sasano $M$, Fuzisawa $M$, Okabe $T$ Nishizawa $K$. Constitutive production of angiotensin converting enzyme from rheumatoid nodule cells under serum free conditions. Ann Rheum Dis 1992; 51: 741-2.

4 Favaloro E J, Moraitis N, Koutts J, Exner T, Bradstock K F. Endothelial cells and normal circulating haemopoietic cells share a number of surface antigens. Thromb Haemost 1989; 61: of surface

5 Hogg N. Surface molecules and receptors. In: Zembala M, Asherson G L, eds. Human monocytes. London: Academic Press, 1989. 37-8.

6 Lipton B H, Bensch K G, Karasek M A Histamine-modulated trans-differentiation of dermal microvascular endothelial cells. Exp Cell Res 1992; 199: 279-91.

7 Fassbender H G, Simmling-Annefeld M. The potential aggressiveness of synovial tissue in potential aggressiveness of synovial tissue in 399-406.

8 McCachren S S. Expression of metaloproteinases and metaloproteinase inhibitor in human arthritic synovium. Arthritis Rheum 1991; 34: 1085-93.

9 Nickoloff B J, Griffiths C E M. The spindleshaped cells in cutaneous Kaposi's sarcoma: histological simulators include factor XIII dermal dendrocytes. Am $\mathcal{F}$ Pathol 1989; 135:

10 Beranek J T. Antibody HAM56 is not macrophage specific in vein autografts. Am $\mathcal{F}$ Cardiol 1991 68: 842

11 Peacock D J, Banquerigo M L, Brahn E. Angiogenesis inhibition suppresses collagen arthritis. F Exp Med 1992; 175: 1135-8.

12 Fernandez L A, Twickler J, Mead A. Neovascularization produced by angiotensin II. $\boldsymbol{f} \mathrm{Lab}$ Clin Med 1985; 105: 141-5.

\section{Endothelium damage and von Willebrand factor antigen}

Sir: We read with great interest the recent paper by Blann et al on damage to the endothelium in Siögren's syndrome. ${ }^{1}$ The authors examined the relation of the autoantibodies SSA and SSB to endothelium damage using serum levels of von Willebrand factor antigen (vWFAg) as an index of damage to the endothelium.

Damage of the endothelium may be present in Siögren's syndrome, but there is no real evidence whether such damage results in high or low levels of vWFAg. The extent of the production of vWFAg in megakaryocytes in Sjögren's syndrome and other diseases is unknown; mainly because the plasma levels of the factor do not follow changes in the numbers of thrombocytes in peripheral blood and so this production site is no longer a part of the scenarium. Further, in our opinion, endothelium damage cannot explain why increased concentrations of VWFAg are present in such varied diseases as proliferative glomerulonephritis, diabetes mellitus, systemic sclerosis, Siögren's syndrome, and arteritis temporalis, as endothelial damage in these diseases is not a common denominator.

We have recently studied the immunohistochemistry in arteritis temporalis, in which 


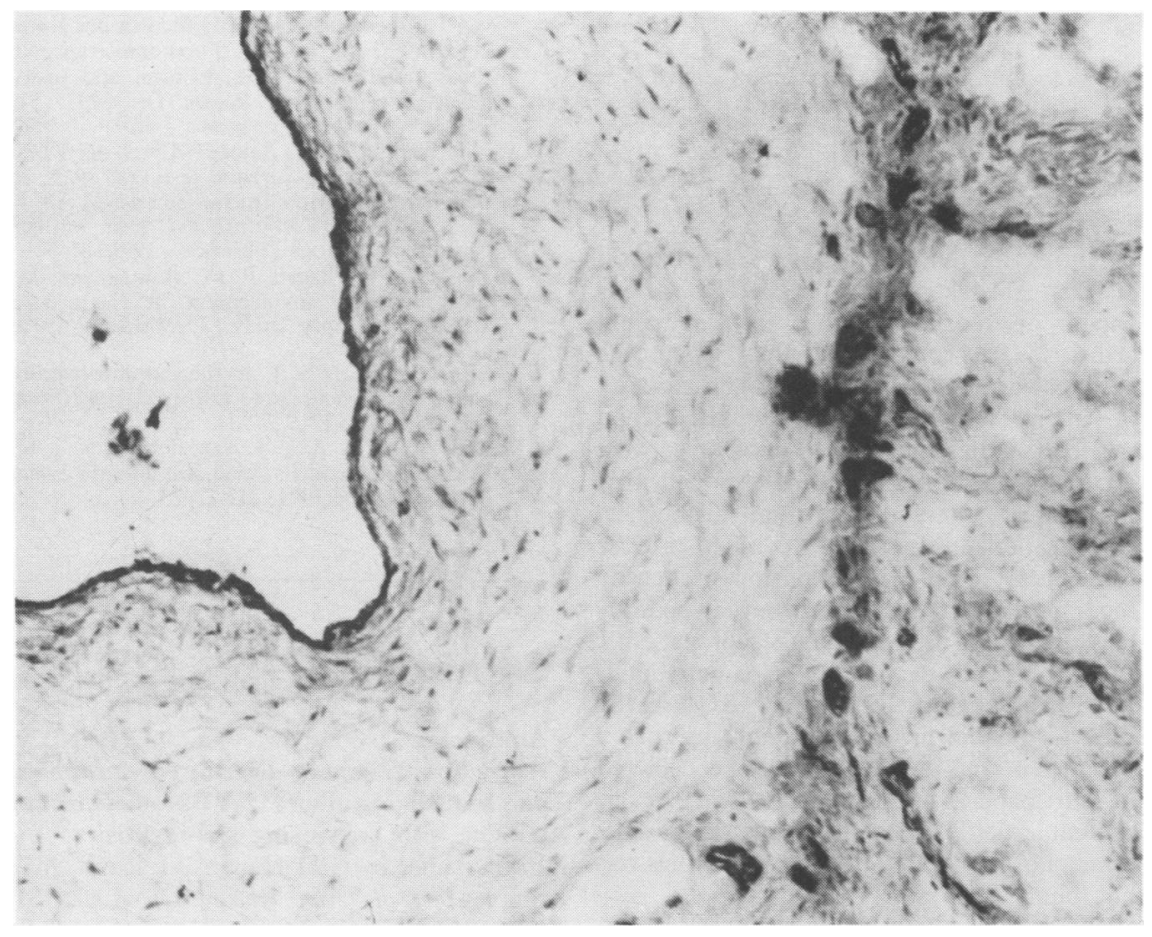

Temporal artery from a patient with arteritis temporalis. Immunoperoxidase and counterstaining with haematoxylin. Von Willebrand factor antigen was localised in endothelial cells of the vessel and in large amounts in new vessels along the lamina elastica.

there are no signs of damage to the endothelium, and have shown ${ }^{2}$ that intense production of vWFAg occurs in the many new vessels occurring in the inflammatory infiltrates (figure). The figure also shows the presence of vWFAg as a slight immunoperoxidase staining in endothelium cells. This staining pattern is not different from the pattern seen in normal temporal arteries or in biopsy specimens from patients with polymyalgia rheumatica and normal levels of vWFAg. As similar microvascular abnormalities (the introduction of many new vessels) are present in most of the diseases with increased $v$ WFAg mentioned above, the reliability of raised levels of VWFAg in these diseases, including Sjögren's syndrome, as a marker of endothelial damage should be reconsidered.

PREBAN ELLING Department of Intermal Medicine
Randers Central Hospital Randers, Denmark

ANDERS OLSSON HANNE ELLING Department of Rheumatology Viborg County Hospital Viborg, Denmark

Correspondence to: Dr Hanne Elling, Department of Rheumatology, Viborg County Hospital, 8800 Viborg, Denmark.

1 Blann A D, Wainwright A C, Emery P. Damage to the endothelium in Siögren's syndrome: lack of correlation with antinuclear antibody titre of correlation with antinuclear antibody titre
and presence of antibodies to SSA and SSB autoantigens. Ann Rheum Dis 1992; 51: autoantigen.

2 Olsson A, Elling P, Elling H. Serological and immunohistochemical determination of von Willebrand factor antigen in serum and biopsy specimens from patients with arteritis temporalis and polymyalgia rheumatica. Clin Exp Rheumatol 1990; 8: 55-8.
AUTHOR'S REPLY: Elling et al propose that raised circulating levels of von Willebrand factor antigen (vWFAg) reflect not endothelial cell damage but production by endothelial cells in newly growing microvasculature, challenging existing dogma. ${ }^{1-7}$ In support of this hypothesis they offer an immunohistological photograph from a patient with temporal arteritis taken from a previous publication which examined temporal artery biopsy specimens from patients with arteritis temporalis, polymyalgia rheumatica, and other disease. ${ }^{8}$ They found raised serum vWFAg and intense vWFAg staining in new vessels in the lamina elastica only in patients with arteritis temporalis, but offer no mechanism or evidence that these are 'new' vessels. Could it be that the intense staining seen in the elastic lamina is because these endothelial cells are damaged? From the photograph it is difficult to tell if all or part of the intense staining for vWFAg is from intact cells, disrupted cells, or from vWFAg in the general connective tissue stroma.

I am surprised that Elling et al suggest that 'endothelium damage cannot explain why increased concentrations of vWFAg are present in such varied diseases as proliferative glomerulonephritis, diabetes mellitus, systemic sclerosis, Sjögren's syndrome, and arteritis temporalis, as endothelial damage in these diseases is not a common denominator'. Other workers, such as Ciompi $e t$ al, have expressed an opposite view, stating that vessel injury is a common feature of scleroderma, glomerulonephritis, diabetes mellitus, Behçet's syndrome, systemic lupus erythematosus, rheumatoid arthritis, and vasculitis in general. ${ }^{5}$

There seems to be little disagreement about the presence of raised vWFAg in a large number of conditions, some of which may be characterised by histological evidence of injury to the vasculature. However, the exact mechanism, in many cases, is unclear. Raised vWFAg is common in the acute phase response, ${ }^{9}$ but it would be surprising if there was evidence of damaged endothelium in this condition. It may be that in this case endothelial cells are merely 'activated' or 'stimulated' to produce $\mathrm{VWFAg}$ in expectation of a more severe insult (i.e. septicaemia), which may indeed damage the endothelium. Further increases in vWFAg may be the product of increased synthesis by actively growing cells in the capillary beds, the adventitia, or elsewhere. If this were true then one would need to explain why there should be an extra growth of endothelial cells, or upregulated production from a resting cell. Perhaps existing cells are being damaged, possibly to the point of cell death, by a disease process, such as the combined effects of immune complexes and complement in vasculitis ${ }^{7}$ or ketoacidosis in diabetes. ${ }^{10}$

Probably the ultimate proof of injury/ damage would be an electron micrograph study of the endothelium in human disease. Such work would need to show a damaged endothelial cell with absent or depleted Wiebel-Palade bodies (the storage organelles of vWFAg), ideally with immunocytochemistry for cytoplasmic vWFAg, alongside plasma levels of vWFAg. Another approach may be to look at vWFAg mRNA from in vivo 'damaged' and 'viable' endothelial cells - but these data would not provide information about vWFAg in Wiebel-Palade bodies.

Until such data are easily obtained then the hypothesis that raised vWFAg is produced by injured endothelial cells in vivo may never be completely accepted. Yet despite this there remains a wealth of clinical and non-clinical data from many different diseases which all support the hypothesis. Convincing competing hypotheses would need to take account of all these findings.

A D BLANN

Department of Surgery, South Mospital of Soll Lame Manchester, Nell Lane, Didsoury, United Kingdom

1 Boneu B, Abbal M, Plante J, Bierme R. FactorVIII complex and endothelial damage. Lancet VIII complex

2 Kahaleh M B, Osborn I, LeRoy E C. Increased factor VII/von Willebrand factor antigen and von Willebrand factor activity in scleroderma and in Raynaud's phenomenon. Ann Intern Med 1981; 94: 482-4.

3 Woolf A D, Wakerley G, Wallington T B, Scott D G I, Dieppe P A. Factor VIII related antigen in the assessment of vasculitis. Ann Rheum Dis 1987; 46: 441-7.

4 Anonymous. Factor VIII related antigen and vasculitis [Editorial]. Lancet 1988; i: 1203-4.

5 Ciompi M L, Marotta G, Puccetti L, et al. Behavior of von Willebrand factor antigen in Behavior of von Willebrand factor antigen in
follow up of polymyalgia rheumatica/giant cell follow up of polymyalgia rheumatica/giant cell
arteritis. Scand $\mathcal{F}$ R heumatol 1988; 17: 491-5.

6 Kahaleh M B. The role of vascular endothelium in the pathogenesis of connective tissue disease: endothelial injury, activation, participation and response. Clin Exp Rheumatol 1990; 8: 595601 .

7 Blann A D. Endothelial cell damage in rheumatoid disease correlates with complement consumption and immune complexes. Rheumacol Int 1991; 11: 87.

8 Olsson A, Elling P, Elling H. Serological and immunohistochemical determination of von Willebrand factor in serum and biopsy von Willebrand factor in serum and biopsy
specimens from patients with arteritis temporalis and polymyalgia rheumatica. Clin Exp poralis and polymyalgia r

9 Pottinger B E, Read R C, Paleolog E M, Higgins $P$ G, Pearson J D. von Willebrand factor is an acute phase reactant in man. Thromb Res 1989; 53: 387-94.

10 Greaves M, Pickering C, Knight G, et al. Changes in the factor VIII complex in diabetic ketoacidosis: evidence of endothelial damage? Diabetologia 1987; 30: 160-5. 